\title{
Satisfação corporal, idade gestacional e estado nutricional em gestantes
}

\section{Body satisfaction, gestational age and nutritional status in pregnant women}

Juliana Fernandes Filgueiras Meireles ${ }^{1}$, Clara Mockdece Neves ${ }^{1}$, Pedro Henrique Berbert de Carvalho ${ }^{2}$, Maria Elisa Caputo Ferreira ${ }^{1}$

\section{RESUMO}

Introdução: A avaliação da imagem corporal durante a gestação é importante para que as gestantes tenham assistência especializada enquanto lidam com as transformações dessa fase. Objetivo: Avaliar a satisfação corporal de gestantes, associar esta à idade gestacional e ao índice de massa corporal (IMC) e comparar grupos por idade gestacional e por estado nutricional quanto à satisfação. Métodos: Participaram 55 gestantes que realizavam pré-natal em um consultório médico particular da cidade de Juiz de Fora, Minas Gerais. Para a avaliação da satisfação corporal foi aplicada a Escala de Medida em Imagem Corporal (EMIC). Foram coletados dados antropométricos (massa corporal e estatura) e obstétricos. As gestantes foram comparadas por estado nutricional e por período gestacional quanto à satisfação corporal. Ademais, buscouse verificar a relação entre a satisfação corporal e o IMC. Resultados: $O$ escore médio e o desvio-padrão da satisfação corporal das gestantes foram de $85,7 \pm 17,98$ pontos. A maioria da amostra estava no segundo trimestre gestacional e com IMC considerado adequado (45 e 38\%, respectivamente). Foram encontradas associações significativas entre a massa corporal e o IMC com a satisfação corporal. Gestantes de baixo peso apresentaram satisfação corporal superiores $(p<0,05)$ em relação às gestantes com sobrepeso e obesidade. Não foram encontradas diferenças para as pontuações da EMIC entre os grupos por estado nutricional e por idade gestacional (primeiro segundo ou terceiro trimestre). Conclusão: Gestantes com menor IMC apresentam maior satisfação corporal, independente da idade gestacional; e ainda, a massa corporal e o IMC são variáveis relacionadas à insatisfação com o corpo.

Palavras-chave: imagem corporal; gravidez; estado nutricional; idade gestacional; adulto.

\begin{abstract}
Introduction: The evaluation of body image during pregnancy is important for pregnant women to have specific assistance while dealing with the changes of that stage. Objective: To evaluate the body satisfaction of pregnant women, to associate this to gestational age and body mass index (BMI), and to compare groups by gestational age and nutritional status regarding body satisfaction. Methods: 55 pregnant women who performed prenatal in a private clinic in Juiz de Fora, Minas Gerais, Brazil took part. For the assessment of body satisfaction, the Escala de Medida de Imagem Corporal (EMIC) was used. Anthropometric and obstetric data were collected. The pregnant women were compared by nutritional status and gestational age in relation to body satisfaction. In addition, the relationship between body satisfaction and BMI was investigated. Results: The mean score and standard deviation for pregnant women's body satisfaction was $85.7 \pm 17.98$ points. The majority of the sample was in the second gestational trimester and BMI was considered adequate (45 and 38\%, respectively). Significant associations between body mass and BMl with body satisfaction were found. Low weight pregnant women showed higher body satisfaction $(p<0.05)$ than pregnant women with overweight and obesity. No differences were found for scores of EMIC between groups by nutritional status and by gestational age (first, second or third trimester). Conclusion: Pregnant women with lower BMI have a higher body satisfaction, regardless of gestational age; and yet, body weight and BMI are variables related to body dissatisfaction.
\end{abstract}

Keywords: body image; pregnancy; nutritional status; gestational age; adults. 


\section{INTRODUÇÃO}

A imagem corporal é a representação do corpo na nossa mente, e está em constante alteração, em permanente construção e desconstrução ${ }^{1}$. Portanto, fatores como sexo, idade, meios de comunicação e aspectos culturais podem influenciar a imagem corporal do indivíduo ${ }^{1,2}$.

$\mathrm{Na}$ sociedade atual, prega-se a cultura do corpo jovem, belo e magro, nem sempre possível de ser alcançado ${ }^{1,2}$. Segundo Dittmar ${ }^{3}$ e Lópes-Guimerà et al. ${ }^{4}$, existe uma forte tendência cultural em considerar a magreza como situação ideal de aceitação para as mulheres. A pressão social e midiática para a adoção do corpo magro enquanto modelo corporal tem forte correlação com o aparecimento da insatisfação corporal, principalmente em jovens adultas do sexo feminino ${ }^{1,2,5}$.

A insatisfação com o próprio corpo é um dos componentes da imagem corporal, relacionada com as atitudes dos indivíduos, e também com as avaliações subjetivas negativas sobre seu corpo ${ }^{1,2}$. Em muitos casos, a avaliação que o indivíduo faz de sua imagem corporal não corresponde à realidade, em que muitas vezes existe uma superestimativa do seu peso e da sua forma, podendo gerar insatisfação corporal ${ }^{6,7}$.

Ao longo da gravidez, as mulheres tendem a reavaliar sua imagem corporal à medida que aumenta seu tamanho e muda a forma do seu corpo ${ }^{8,9}$. As rápidas mudanças corporais que ocorrem no período gestacional afastam as gestantes do ideal de corpo propagado e valorizado para as mulheres, podendo, portanto, ocasionar um sentimento de descontentamento com o corpo e com sua imagem ${ }^{8-11}$.

A gravidez consiste em um processo fisiológico natural de desenvolvimento do embrião dentro do organismo feminino e compreende uma sequência de adaptações ocorridas a partir da fertilização ${ }^{12,13}$. Durante todo o processo, a gestante atravessará transformações que repercutem de forma expressiva no seu dia a dia, que são de fundamental importância e têm como objetivo proporcionar condições para o adequado desenvolvimento fetal em equilíbrio com o organismo materno ${ }^{13}$.

Nesse momento da vida da mulher ocorre um aumento do peso e do tamanho corporal em um período de tempo relativamente curto ${ }^{8,9}$. No entanto, apesar de naturais, as mudanças corporais ocorridas durante a gravidez dificultam a acomodação da imagem corporal ${ }^{8}$.

Skouteris et al. ${ }^{9}$ ressaltam a importância da avaliação da imagem corporal nesse período para que as gestantes tenham assistência especializada, enquanto elas lidam com as transformações típicas dessa fase da vida da mulher. Entretanto, ainda são escassos na literatura brasileira estudos que avaliem a satisfação corporal nos diferentes períodos da gestação ${ }^{14}$

Dessa forma, o presente estudo teve como objetivo avaliar a satisfação corporal de gestantes, além de verificar possíveis associações entre a satisfação corporal e as variáveis independentes (idade gestacional, massa corporal e índice de massa corporal - IMC) e comparar a satisfação corporal de gestantes entre os grupos por idade gestacional e por estado nutricional.

\section{MÉTODOS}

Este estudo foi aprovado pelo Comitê de Ética para pesquisa em Seres Humanos da Universidade Federal de Juiz de Fora (UFJF), sob o protocolo 2419.159.2011 (Parecer no 169/2011). Todas as gestantes foram informadas sobre os objetivos e riscos da pesquisa e concordaram em participar por meio da assinatura do Termo de Consentimento Livre e Esclarecido (TCLE).

Quando há poucos conhecimentos sobre os objetos de estudos, recomenda-se a realização de um estudo exploratório dos temas. Dessa forma, este estudo caracteriza-se como do tipo exploratório e transversal com uma amostra por conveniência ${ }^{15}$. Participaram 55 gestantes que realizavam o pré-natal em um consultório médico particular da cidade de Juiz de Fora, Minas Gerais, durante a fase de coleta de dados.

Foram incluídas gestantes em todas as idades gestacionais, maiores de 18 anos, que soubessem ler e escrever, e que aceitaram participar voluntariamente da pesquisa. Foram excluídas gestantes que por algum motivo não participaram de alguma etapa da coleta de dados, que apresentaram dados incompletos, ou ainda com gravidez considerada de risco (como, por exemplo, hipertensas, cardiopatas, soropositivas, de gravidez múltipla, diabéticas) de acordo com o seu prontuário.

Para a avaliação da satisfação corporal foi utilizada a Escala de Medida em Imagem Corporal (EMIC), elaborada para a população brasileira por Souto ${ }^{16}$ e utilizada anteriormente em uma amostra de gestantes ${ }^{17}$. A escala é composta por 23 questões, sendo 5 com significado favorável e 18, desfavorável. As questões estão dispostas como uma escala do tipo Likert, de 1 (nunca) a 5 pontos (sempre), em que o indivíduo aponta com que frequência pensou, se sentiu, ou se comportou diante dos eventos propostos pelas alternativas. Os escores são obtidos pela soma das 23 questões e variam de 23 e 115 pontos. Esse instrumento não apresenta ponto de corte, entretanto, pontuações altas são indicativas de uma imagem corporal favorável (satisfação corporal) e, ao contrário, pontuações baixas são indicativas de alteração de imagem corporal.

A consistência interna geral do instrumento original, obtida por meio do alpha de Cronbach, foi de $0,91^{16}$, valor similar ao encontrado na versão utilizada em gestantes $(\alpha=0,912)$ por Cairolli e Shimo ${ }^{17}$. Segundo Hair et al. ${ }^{18}$, a confiabilidade do instrumento, analisada por meio do alpha de Cronbach, deve assumir valores maiores do que 0,70, e devem ser testada a fim de se garantir a validade interna do instrumento. Para a amostra deste estudo, o instrumento apresentou alpha de Cronbach de 0,94 , confirmando sua confiabilidade.

Os dados antropométricos foram coletados sempre pelo mesmo médico responsável, especialista em Ginecologia e Obstetrícia, no próprio consultório, considerado espaço adequado para as medições. A medida da massa corporal foi realizada utilizando uma balança da marca Filizola ${ }^{\circledR}$, com precisão de 100 g, devidamente aferida e com o selo do INMETRO. Em seguida, foi feita a medida da estatura, por meio de um estadiômetro de aço da marca WCS com precisão de 1 milímetro $(\mathrm{mm})$. 
Para a classificação do estado nutricional, utilizou-se o IMC. Foi utilizada a classificação a partir de quatro grupos, levando em consideração a massa corporal e a estatura de acordo com a semana gestacional, como proposto para essa população ${ }^{13,19}$, em que são considerados: baixo peso, adequado, sobrepeso e obesidade. Da mesma forma, os trabalhos sobre medidas de satisfação com a imagem corporal têm utilizado o IMC como indicador do estado nutricional ao associá-lo com fatores determinantes das condutas relativas ao $\operatorname{corpo}^{20}$.

Através dos prontuários das gestantes, preenchidos pelo próprio médico no dia das consultas de exame pré-natal, foram obtidas as informações sobre a idade gestacional (semanas) e os dados sobre a saúde materna e do bebê (presença de hipertensão, cardiopatias, gravidez múltipla, diabetes, e infecção viral por HIV).

Este estudo foi realizado no próprio consultório do médico responsável, localizado em um bairro central da cidade de Juiz de Fora, Minas Gerais. As participantes foram convidadas a participar da pesquisa voluntariamente, nos dias das consultas de exame de pré-natal, anteriormente ao seu atendimento. Em caso de consentimento, foram esclarecidas sobre os procedimentos da pesquisa e assinaram o TCLE. Posteriormente, foi entregue o questionário de avaliação da satisfação corporal.

Nesse mesmo dia, o médico realizou a aferição da massa corporal e estatura das pacientes com anotação dos dados no prontuário da paciente, juntamente aos dados obstétricos, que foram analisados posteriormente.

\section{Análise dos dados}

Para a análise dos dados foi utilizado o software estatístico Statistica versão 8.0. Foram realizadas análises descritivas dos dados (idade, idade gestacional, medidas antropométricas, EMIC) com medidas de tendência central (média) e de dispersão (desvio padrão, valores mínimo e máximo). A distribuição das gestantes quanto à idade gestacional e às classificações do IMC foram descritas em frequência absoluta e relativa. A estatística inferencial foi aplicada para relatar a consistência interna ( $\alpha$-Cronbach) do questionário de satisfação corporal.

Foi aplicado teste de normalidade Kolmogorov-Smirnov para análise das características dos dados. Para as associações das variáveis foi utilizado teste de associação de Pearson. Para as comparações entre os grupos de estado nutricional (baixo peso, adequado, sobrepeso e obesidade) e as idades gestacionais (primeiro trimestre, segundo trimestre e terceiro trimestre) quanto à satisfação corporal, foi utilizado o teste de comparação ANOVA one-way, seguido pelo Post-hoc de Scheffé.

Para todas as análises estatísticas foi adotado o nível de significância de $5 \%$.

\section{RESULTADOS}

Participaram da pesquisa 55 gestantes com média de $30,6 \pm 5,95$ anos, apresentando valores no EMIC, variando de 31 pontos (mínimo) a 111 pontos (máximo), como observado na Tabela 1.

Com relação à idade gestacional, 22\% (12) das investigadas estavam no primeiro trimestre, $45 \%$ (25) no segundo e $33 \%$ (18) no terceiro. Para classificação quanto ao estado nutricional, foi encontrado que $20 \%$ (11) das gestantes estavam com baixo do peso, $38 \%$ (21) foram consideradas com o peso adequado, $24 \%$ (13) com sobrepeso e $18 \%$ (10) com obesidade.

Verificou-se correlação negativa e estatisticamente significativa entre a massa corporal e EMIC, bem como para EMIC e IMC; ou seja, as gestantes com maior massa corporal e IMC apresentaram menores valores para satisfação corporal (Tabela 2).

Quando comparados os grupos por idade gestacional quanto à satisfação (EMIC), não foi encontrada diferença estatisticamente significativa entre os grupos (Figura 1).

Tabela 1: Análise descritiva das variáveis: idade, massa corporal, estatura, índice de massa corporal, idade gestacional e Escala de Medida em Imagem Corporal

\begin{tabular}{|l|c|c|c|c|c|c|}
\hline Variáveis & $\mathbf{n}$ & Média & Desvio padrão & Mediana & Mínimo & Máximo \\
\hline Idade (anos) & 55 & 30,6 & 5,95 & 30,00 & 20,00 & 44,00 \\
\hline Massa corporal $(\mathrm{kg})$ & 55 & 69,6 & 14,85 & 65,10 & 46,00 & 115,90 \\
\hline Estatura $(\mathrm{m})$ & 55 & 1,6 & 0,06 & 1,61 & 1,50 & 1,76 \\
\hline IMC $\left(\mathrm{kg} / \mathrm{m}^{2}\right)$ & 55 & 26,7 & 5,47 & 26,08 & 17,93 & 45,27 \\
\hline Idade gestacional (semanas) & 55 & 21,9 & 9,27 & 22,00 & 6,00 & 39,00 \\
\hline EMIC & 55 & 85,7 & 17,98 & 89,00 & 31,00 & 111,00 \\
\hline
\end{tabular}

IMC: índice de massa corporal; EMIC: Escala de Medida em Imagem Corporal.

Tabela 2: Associação entre as variáveis (Escala de Medida em Imagem Corporal, índice de massa corporal, massa corporal, idade e idade gestacional)

\begin{tabular}{|l|c|c|c|c|c|}
\hline Variáveis & EMIC & IMC & Massa corporal & Idade & \\
\hline EMIC & 1,00 & & & \\
\hline IMC $\left(\mathrm{kg} / \mathrm{m}^{2}\right)$ & $-0,50^{*}$ & 1,00 & & \\
\hline Massa corporal $(\mathrm{kg})$ & $-0,48^{*}$ & $0,94^{*}$ & 1,00 & 1,00 \\
\hline Idade (anos) & 0,12 & 0,13 & 0,14 & $-0,07$ & 1,00 \\
\hline Idade gestacional (semanas) & $-0,73$ & $0,33^{*}$ & $0,26^{*}$ & \\
\hline
\end{tabular}

EMIC: Escala de Medida em Imagem Corporal; IMC: índice de massa corporal; *significativo para $p<0,05$. 
A comparação entre os grupos por estado nutricional quanto ao EMIC apresentou diferença estatisticamente significativa; $\mathrm{F}(3,51)=6,59 ; \mathrm{p}=0,0007$ (Figura 2). As análises demonstraram diferenças significativas entre os grupos de baixo peso e sobrepeso $(100,55$ versus 76,$23 ; \mathrm{p}=0,005)$ e baixo peso e obesidade $(100,55$ versus 75,$20 ; \mathrm{p}=0,006)$.

\section{DISCUSSÃO}

O presente estudo buscou avaliar a satisfação corporal em gestantes da cidade de Juiz de Fora, Minas Gerais, bem como verificar possíveis associações entre essa variável e a idade gestacional, massa corporal e IMC. Além disso, procurou-se comparar a satisfação corporal entre os grupos por idade gestacional e por

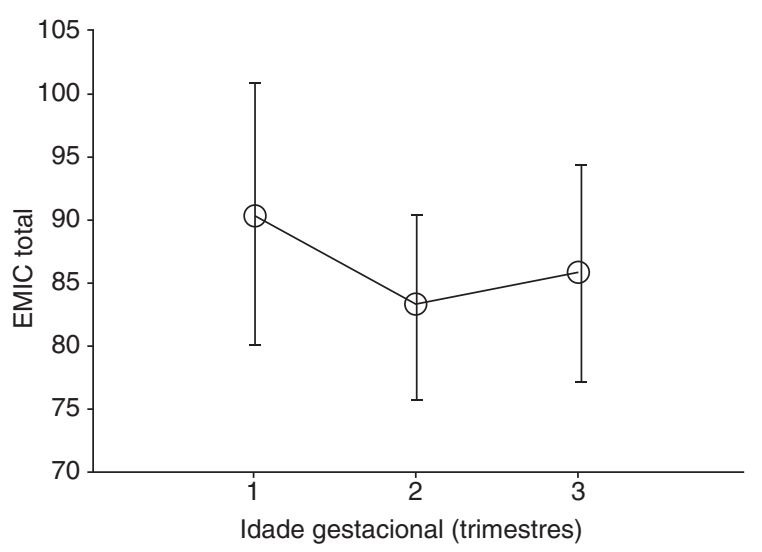

EMIC: Escala de Medida em Imagem Corporal.

Figura 1: Comparação entre os grupos por idade gestacional quanto à satisfação corporal (Escala de Medida em Imagem Corporal)

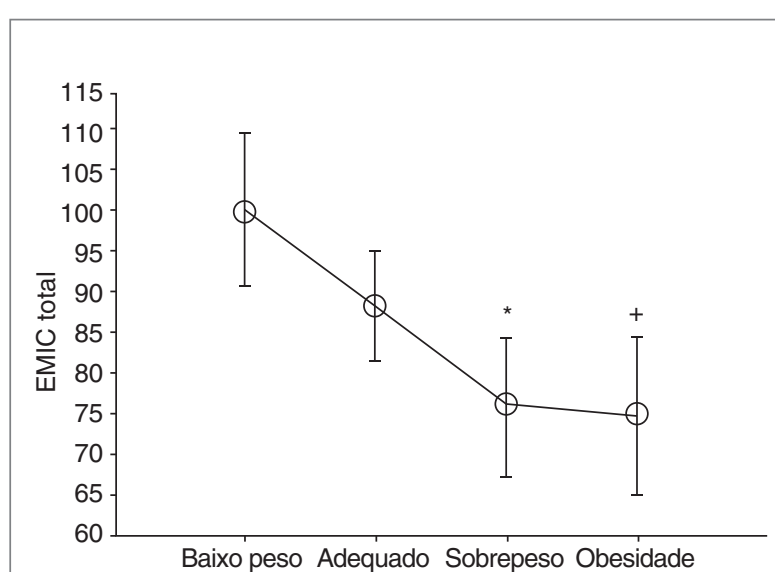

Baixo peso Adequado Sobrepeso Obesidade

EMIC: Escala de Medida em Imagem Corporal; *diferença significativa entre baixo peso e sobrepeso; +diferença significativa entre baixo peso e obesidade.

Figura 2: Comparação entre Escala de Medida em Imagem Corporal e estado nutricional estado nutricional. De acordo com os achados da literatura, são escassos, no Brasil, estudos que investigaram a insatisfação com a imagem corporal em gestantes relacionando-a a outras variáveis ${ }^{14}$. Os resultados desta pesquisa exploratória destacam que algumas variáveis estão significativamente associadas à imagem corporal de gestantes.

A partir dos dados descritivos, verificou-se uma média de idade das gestantes que pode ser considerada alta (aproximadamente 30 anos). A faixa de idade considerada ideal para engravidar é dos 19 aos 32 anos $^{13}$, sendo que, a partir dos 35 anos, no caso de primigestação, a gravidez é considerada de alto risco ${ }^{13}$. Em um estudo realizado por Cairolli ${ }^{21}$, 47,31\% das gestantes se encontravam na faixa etária de 19 a 25 anos. A amostra estudada apresentou uma idade tardia para a gestação, o que segue uma tendência atual das mulheres engravidarem mais tardiamente.

Quanto ao estado nutricional, a maioria da amostra foi considerada com o peso adequado (38\%), porém, foi alto o número de gestantes com baixo (20\%) e alto (18\%) IMC quando comparadas à população feminina adulta ${ }^{22}$. Vale ressaltar que as gestantes com IMC inadequado deste estudo não apresentavam problemas associados à gravidez. A importância de se manter no peso adequado durante a gestação, juntamente com uma alimentação adequada, deve ser enfatizada para essas mulheres, prevenindo possíveis problemas de saúde que refletem não só na saúde da mãe, como também na saúde fetal ${ }^{14,20}$.

Com relação à satisfação corporal, foi encontrado um escore médio de 85,7 pontos, variando de 31 (mínimo) a 111 pontos (máximo). Os achados de Cairolli21 ${ }^{21}$ utilizando a mesma escala, demonstraram valores próximos (média de 95, mínimo de 39 e máximo de 115 pontos). Apesar do instrumento não apresentar classificações para a satisfação corporal, a pontuação obtida pela amostra do presente estudo contempla quase a totalidade de variação possível do instrumento (23 a 115 pontos). Isso pode demonstrar que a variável em questão oscilou consideravelmente, não apresentando um padrão entre as gestantes estudadas.

A satisfação corporal apresentou correlação negativa com a massa corporal e o IMC, ou seja, gestantes com maiores IMC e massa corporal apresentaram menores escores no EMIC, indicando menor satisfação corporal. A relação entre essas variáveis já foi amplamente discutida na literatura em diversas amostras ${ }^{7,23,24}$. Em gestantes, segundo um estudo realizado na Austrália, a insatisfação corporal está relacionada à massa corporal adquirida ${ }^{10}$. Os primeiros estudos nacionais em gestantes também demonstraram uma associação entre o IMC e a insatisfação corporal ${ }^{17,20,21}$. As mulheres que apresentam seu IMC elevado tendem à insatisfação e valorizam o corpo de mulheres com IMC mais baixo. Assim, as grávidas são influenciadas pela magreza, considerada ideal corporal atual, assim como as mulheres não grávidas ${ }^{6,14}$.

As gestantes com sobrepeso e obesidade apresentaram maior insatisfação corporal quando comparadas às de baixo peso. No entanto, esses grupos não apresentaram diferenças significativas em relação às grávidas com IMC adequado. Vale ressaltar que, 
apesar do grupo de baixo IMC ter apresentado maiores índices de satisfação, esse estado nutricional é considerado inadequado para a saúde materna e do bebê.

Na presente pesquisa não foi identificada diferença nos escores de satisfação corporal entre os grupos por idade gestacional. Uma das hipóteses é que a satisfação corporal seria diferente entre os grupos, assim como apontam Skouteris et al. ${ }^{9}$, pois o ganho de peso que acontece gradativamente durante a gravidez estaria modulando a satisfação. No entanto, Duncombe et al. ${ }^{25}$ observaram associação entre o período gestacional e o IMC, e não verificaram diferença na satisfação entre os grupos. Possivelmente, os diferentes achados da literatura quanto à satisfação corporal durante o período gestacional pode ocorrer pela modulação de outras variáveis não avaliadas neste estudo, como por exemplo, a prática de atividade física, as relações familiares e sociais, o apoio do cônjuge e outras, apontadas por Cairolli ${ }^{21}$ como importantes para essa população.

Este estudo apresenta algumas limitações que merecem destaque. Inicialmente, o desenho de corte transversal impossibilita a verificação da relação de causa e efeito entre as variáveis. No entanto, pela carência de estudos nacionais sobre o tema ${ }^{14}$, e considerando o resultado das análises estatísticas que corroboram outros estudos internacionais, acredita-se que esse possa ser um importante contributo para a ampliação do entendimento sobre a satisfação corporal de gestantes brasileiras.

Outro fato importante é a inexistência de instrumentos criados e validados no Brasil, especificamente para essa população, o que dificulta a utilização de mais de um instrumento para avaliar um construto complexo como a imagem corporal. Thompson ${ }^{26}$ alerta para essa necessidade metodológica, a fim de que as medidas possam ser consideradas válidas e confiáveis. Este estudo valeu-se da EMIC, escala criada e validada para população feminina adulta brasileira ${ }^{16}$; e já utilizada anteriormente por Cairolli e Shimo ${ }^{17}$ para gestantes brasileiras, demonstrando adequados valores de validade inicial. Foram tomados os cuidados metodológicos apontados por Hair et al. ${ }^{18}$, com o cálculo da consistência interna do instrumento para nossa amostra, apresentando adequado valor de alpha de Cronbach, indicando a validade de medida. Por fim, outra limitação se deve ao fato de não terem sido coletados dados econômicos e sociodemográficos, impossibilitando uma descrição mais detalhada da amostra.

Novos estudos ainda são necessários na tentativa de associar variáveis, tais como: nível socioeconômico, autoestima, níveis de estresse e depressão, satisfação corporal pré-gestacional e comportamento alimentar. Estudos longitudinais podem ampliar o entendimento sobre o tema, possibilitando intervenções futuras sobre a saúde e qualidade de vida das gestantes e sua influência sobre a saúde do bebê.

Conclui-se que há associação entre a satisfação corporal de gestantes e massa corporal e o IMC. Além disso, gestantes com baixo IMC apresentam maior satisfação com o corpo do que aquelas com sobrepeso e obesidade, não sendo encontrada diferença entre as idades gestacionais.

Por se tratar de uma fase de muitas peculiaridades, na qual as mulheres passam por inúmeras mudanças em seus corpos, a gravidez é um momento em que as intervenções para tratar excesso de peso/obesidade e insatisfação corporal têm grandes chances de serem bem sucedidas. A prevenção de altos níveis de insatisfação com o corpo só será eficaz quando os modelos de fatores de risco foram examinados de forma sistemática e rigorosa, a fim de uma maior compreensão.

\section{AGRADECIMENTOS}

A todas as gestantes que se disponibilizaram a participar desta pesquisa e ao Laboratório de Estudos do Corpo (LABESC), pela disponibilização dos instrumentais do estudo.

\section{REFERÊNCIAS}

1. Ferreira MEC, Castro MR, Morgado FFR. Imagem corporal: reflexões, diretrizes e práticas de pesquisa. Juiz de Fora: Editora UFJF; 2014.

2. Cash TF, Smolak L. Body image: a handbook of science, practice, and prevention. 2nd ed. New York: The Guilford Press; 2011.

3. Dittmar H. How do "body perfect" ideals in the media have a negative impact on body image and behaviors? Factors and processes related to self and identity. J Soc Clin Psychol. 2009;28(1):1-8. http://dx.doi.org/10.1521/jscp.2009.28.1.1

4. Lopes-Guimerà G, Levine MP, Sánchez-Carracedo D, Fauquet J. Influence of mass media on body image and eating disordered attitudes and behaviors in females: a review of effects and processes. Media Psychol. 2010;13(4):387-416. http://dx.doi.org/10.1080/15213269.2010.525737

5. Amaral AC, Ribeiro MS, Conti MA, Ferreira CS, Ferreira MEC Psychometric evaluation of the Sociocultural Attitudes Towards
Appearance Questionnaire-3 among Brazilian young adults. Span J Psychol. 2013;16:E94.

http://dx.doi.org/10.1017/sjp.2013.94

6. Teixeira FA, Schneider VLP, Wolpe RE, Sperandio FF. Satisfação com a imagem corporal em mulheres gestantes e não gestantes. ABCS Health Sci. 2015;40(2):69-74.

http://dx.doi.org/10.7322/abcshs.v40i2.733

7. Miranda VPN, Filgueiras JF, Neves CM, Teixeira PC, Ferreira MEC. Insatisfação corporal em universitários de diferentes áreas de conhecimento. J Bras Psiquiatr. 2012;61(1):25-32. http://dx.doi.org/10.1590/S0047-20852012000100006

8. Watson B, Fuller-Tyszkiewicz M, Broadbent J, Skouteris H. The meaning of body image experiences during the perinatal period: a systematic review of the qualitative literature. Body Image. 2015;14(3):102-13.

http://dx.doi.org/10.1016/j.bodyim.2015.04.005 
9. Skouteris H, Carr R, Wertheim EH, Paxton SJ, Duncombe D. A prospective study of factors that lead to body image dissatisfaction during pregnancy. Body Image. 2005;2(4):347-61. http://dx.doi.org/10.1016/j.bodyim.2005.09.002

10. Clark A, Skouteris H, Wertheim EH, Paxton SJ, Milgrom J. The relationship between depression and body dissatisfaction across pregnancy and the postpartum: a prospective study. J Health Psychol. 2009;14(1):27-35. http://dx.doi.org/10.1177/1359105308097940

11. Kamysheva E, Skouteris H, Wertheim EH, Paxton SJ, Milgrom J. Examination of a multi-factorial model of body-related experiences during pregnancy: the relationships among physical symptoms, sleep quality, depression, self-esteem, and negative body attitudes. Body Image. 2008;5(2):152-63.

http://dx.doi.org/10.1016/j.bodyim.2007.12.005

12. Mann L, Kleinpaul JF, Mota CB, Santos SG. Alterações biomecânicas durante o período gestacional: uma revisão. Motriz. 2010;16(3):730-41

http://dx.doi.org/10.5016/1980-6574.2010v16n3p730

13. Brasil. Ministério da Saúde. Gestação de alto risco: manual técnico. Brasília: Editora MS; 2012.

14. Meireles JFF, Neves CM, Carvalho PHB, Ferreira MEC. Insatisfação corporal em gestantes: uma revisão integrativa da literatura. Ciênc Saúde Coletiva. 2015;20(7):2091-2103. http://dx.doi.org/10.1590/1413-81232015207.05502014

15. Thomas JR, Nelson JK, Silverman SJ. Métodos de pesquisa em atividade física. 6ª ed. São Paulo: Artmed; 2012.

16. Souto CMRM. Construção e validação de uma escala de medida em imagem corporal. Dissertação (Mestrado) - Universidade Estadual da Paraíba, João Pessoa, 1999

17. Cairolli PB, Shimo AKK. Análise da consistência interna pelo coeficiente alfa de Cronbach da Escala de Medida em Imagem Corporal aplicada a uma população de gestantes. In: I Simpósio Internacional de Imagem Corporal. I Congresso Brasileiro de Imagem Corporal. Campinas: UNICAMP; 2010.
18. Hair JF, Tatham RL, Anderson RE, Black W. Análise multivariada de dados. $6^{a}$ ed. São Paulo: Bookman; 2009.

19. Rasmussen KM, Yaktine AL. Institute of Medicine and National Research Council Committee to Reexamine IOM Pregnancy Weight Guidelines. Weight gain during pregnancy: reexamining the guidelines. Washington: National Academies Press; 2009.

20. Meireles JFF, Neves CM, Carvalho PHB, Ferreira MEC Imagem corporal de gestantes: associação com variáveis sociodemográficas, antropométricas e obstétricas. Rev Bras Ginecol Obstet. 2015;37(7):319-24.

http://dx.doi.org/10.1590/SO100-720320150005388

21. Cairolli PB. Avaliação da imagem corporal e da (in)satisfação com o corpo grávido pela escala de medida em imagem corporal em gestantes inscritas no programa de pré-natal da rede básica de saúde de Vinhedo - SP. Dissertação (Mestrado) - Universidade Estadual de Campinas, Campinas, 2009.

22. Ferreira VA, Magalhães R. Obesidade no Brasil: tendências atuais. Rev Port Saúde Pública. 2006;24(2):71-82.

23. Miranda VPN, Conti MA, Bastos R, Ferreira MEC. Insatisfação corporal em adolescentes brasileiros de municípios de pequeno porte de Minas Gerais. J Bras Psiquiatr. 2011:60(3):190-7. http://dx.doi.org/10.1590/S0047-20852011000300007

24. Fortes LS, Almeida SS, Ferreira MEC. Indicadores antropométricos de insatisfação corporal e de comportamentos alimentares inadequados em jovens atletas. Rev Bras Med Esporte. 2013;19(1):35-9.

http://dx.doi.org/10.1590/S1517-86922013000100007

25. Duncombe D, Wertheim EH, Skouteris H, Paxton SJ, Kelly L. How well do women adapt to changes in their body size and shape across the course of pregnancy? J Health Psychol. 2008;13(4):503-15. http://dx.doi.org/10.1177/1359105308088521

26. Thompson JK. The (mis)measurement of body image: ten strategies to improve assessment for applied and research purposes. Body Image. 2004;1(1):7-14 http://dx.doi.org/10.1016/S1740-1445(03)00004-4 